
\title{
25 Research Suare \\ Birt-Hogg-Dubé Syndrome Encountered At Rare Lung Disease Clinic In Anhui Province, China
}

Guofeng Zhang

The First Affiliated Hospital of USTC: Anhui Provincial Hospital

Jinli Liu

The First Affiliated Hospital of USTC: Anhui Provincial Hospital

\section{Yushuo Wang}

The First Affiliated Hospital of USTC: Anhui Provincial Hospital

\section{Yue Wang}

The First Affiliated Hospital of USTC: Anhui Provincial Hospital

\section{Xianliang Jiang}

The First Affiliated Hospital of USTC: Anhui Provincial Hospital

\section{Yan Peng}

The First Affiliated Hospital of USTC: Anhui Provincial Hospital Jun Xiao

The First Affiliated Hospital of USTC: Anhui Provincial Hospital

\section{Wei Wei}

The First Affiliated Hospital of USTC: Anhui Provincial Hospital

\section{Bing Shen}

Anhui Medical University

\section{Long Yi}

Nanjing University

Jay H. Ryu

Mayo Clinic Hospital

Xiaowen Hu ( $\nabla$ hu.xiaowen@hotmail.com )

The First Affiliated Hospital of USTC: Anhui Provincial Hospital https://orcid.org/0000-0002-3727-6049

\section{Research Article}

Keywords: Birt-Hogg-Dubé syndrome, FLCN gene, Pneumothorax.

Posted Date: March 8th, 2022

DOI: https://doi.org/10.21203/rs.3.rs-1319752/v1 
License: (c) (i) This work is licensed under a Creative Commons Attribution 4.0 International License. Read Full License 


\section{Abstract}

Background: Diagnosis of rare diseases remains a challenge in China. We describe our experience with Birt-Hogg-Dubé syndrome (BHDS) encountered at a Rare Lung Disease Clinic recently established in China.

Methods: After the first patient with BHDS was recognized in 2017, a Rare Lung Disease Clinic with a multidisciplinary team of specialists was established. We retrospectively analyzed the data of consecutive patients with BHDS encountered from inception to December 2021.

Results: There were 1, 1, 15, 12 and 21 cases with BHDS diagnosed from year 2017 to 2021, respectively. All 50 patients ( 34 women) were of Han race with a mean age of 47.3 years. The common manifestations were pulmonary cysts (98\%), pneumothorax (54\%) and skin lesions (68\%). Renal cancer was detected in two patients and renal angiomyolipoma in four other patients. The main presentations leading to diagnosis were pneumothorax (42\%), family screening (36\%) and lung cysts identified on radiologic imaging $(20 \%)$. The average delay in diagnosis was 8.3 years, and 4.7 years in patients with only pulmonary cysts. The most frequent pathogenic variant was c.1285del/dup on exon 11 (23\%) among 44 patients confirmed by genetic testing. Renal cancer has not been found on follow-up surveillance thus far.

Conclusions: There has been increasing number of patients with BHDS recognized in China, facilitated by establishment of a Rare Lung Disease Clinic. Pulmonary cysts and pneumothorax were commonly encountered features, but skin lesions appeared to be more prevalent in Chinese subjects than previously reported in other Asian countries.

\section{Introduction}

Birt-Hogg-Dubé syndrome (BHDS) is a rare autosomal dominant disorder caused by pathogenic variants in the FLCN gene located on chromosome 17p11.2(1). The disease is characterized by pulmonary cysts often leading to recurrent spontaneous pneumothorax, cutaneous fibrofolliculomas, and an increased risk of renal cell cancers(2). The earliest families of BHDS were reported by Hornstein and Knickenberg in 1975(3) and three Canadian physicians in 1977(4). In the past 40 years, over 600 families have been reported worldwide.

The prevalence of BHD in the general population has been estimated to be 1.86 cases per million(5). Therefore, the calculated number of patients with BHDS in the Chinese population (1.4 billion) is 2604 . In 2008, the first study of BHDS in China was reported by a research team in Nanjing University, China(6). By the end of 2021, there were only 221 Chinese patients reported in the literature(7), which is less than $10 \%$ of potential BHDS population in China. The average delay from the first symptom to final diagnosis of BHDS has been reported to be 13 years in America and 10 years in China $(7,8)$. Misdiagnosis and delayed diagnosis present critical challenges for Chinese patients with BHDS, especially in areas beyond Beijing and Jiangsu Province(7). Previous reports suggested a lower prevalence of skin lesions among of 
BHDS patients in Eastern Asian $(9,10)$. However, the skin lesions were recently found in almost $50 \%$ of confirmed cases from Peking Union Medical College Hospital (PUMCH), a national referred center (11).

This study aims to clarify the characteristics, including the prevalence of skin lesions, in patients with BHDS in Anhui Province, China.

\section{Methods}

A 45-year-old female presenting recurrent pneumothorax and family history of pneumothorax was diagnosed to have BHDS in 2017(12). To improve the diagnosis and management of patients with rare lung diseases such as BHDS, a Rare Lung Disease Clinic was established by Dr. XH at our hospital in June 2019. Based on previous studies on diffuse cystic lung diseases(13-15), suspected patients were initially assessed by an experienced pulmonary physician (Dr. XH) in our Rare Lung Disease Clinic and was supported by a multidisciplinary team of specialists including a radiologist (WW), pathologist (YP), dermatologist $(\mathrm{JL})$, urologist $(\mathrm{JX})$ and thoracic surgeon $(\mathrm{XJ})$. When clinical presentation combined with radiological features of pulmonary cysts were suggestive of BHDS, genetic testing was undertaken after patients' informed consent. The patients who declined participation and whose diagnosis remained unclear were excluded in this study.

A total of 50 consecutive patients who received medical care at our hospital and were diagnosed with BHDS from January 2017 to December 2021 were enrolled into this study. The diagnosis of BHDS was based on the criteria proposed by the European BHD consortium. BHDS was diagnosed by fulfilling one major criterion or two minor criteria(16). The major criteria included: (1) at least five fibrofolliculomas or trichodiscomas (at least one histologically confirmed, of adult onset); (2) pathogenic FLCN germline mutation. The minor criteria included: (1) multiple lung cysts (bilateral basally located lung cysts with no other apparent cause, with or without spontaneous pneumothorax); (2) renal cancer (early onset before age 50 or multifocal or bilateral, or mixed chromophobe and oncocytic histology); (3) a first-degree relative with BHDS.

Clinical information including sex, age, family history, pulmonary cysts, detailed history of pneumothorax, skin lesion, renal tumor, genetic testing, diagnosis, treatment, and follow-up was collected.

Chest computed tomography (CT) studies of each patient were assessed by two pulmonary physicians $(\mathrm{XH}, \mathrm{GZ}$ ) independently for the presence of pneumothorax, cysts and cyst localization(upper or lower dominant; central or peripheral dominant), cyst number $(\varangle 10,10-20, \varangle 20)$, cyst size (maximum diameter: $<1 \mathrm{~cm}, 1-2 \mathrm{~cm}, 2-5 \mathrm{~cm},>5 \mathrm{~cm}$ ), and cyst shape (round to oval, irregular). CT, ultrasound or magnetic resonance imaging of the abdomen was used for renal assessment. Skin lesions were diagnosed by two physicians specialized in rare respiratory diseases $(\mathrm{XH}, \mathrm{GZ})$ and an experienced dermatologist (JL). In cases of discrepancies between the three observers, the differences were resolved by discussion and consensus. Family history of pneumothorax was defined as spontaneous pneumothorax in at least 2 family members, including patients, within three generations. FLCN gene detection was carried out based on methods described in our previous study(17). Genomic DNA was 
extracted from peripheral blood leucocytes according to standard procedures and subjected to polymerase chain reaction and Sanger sequencing. The sequencing reactions were conducted on an $A B I$ 3730XL DNA Analyzer. DNA Sequencing Analysis and Sequencing Analysis 5.2.0 software was used to analyze the sequencing results and using Sequencer 5.1 software package for comparative analysis. When the mutation could not be detected by direct sequencing, MLPA analysis and rapid NGS strategy of the FLCN gene were used based on our experts' judgements (Prof. Shen and Dr. Yi)(18).

All data are expressed in the form of means and standard deviations ( $\mathrm{X} \pm \mathrm{s}$ ). SPSS 25.0 was used for data analysis and independent sample t-test was used to compare continuous variables. Chi-square test was used to compare categorical variables. Differences with a value of $\mathrm{P}<0.05$ was considered statistically significant. This single-center and observational study was approved by the Institutional Review Board at the First Affiliated Hospital of University of Science and Technology (Number $2021 \mathrm{KY} 187)$, and patient consent was signed at the medical service.

\section{Results}

Fifty patients from 31 families were diagnosed with BHDS at our hospital from January 1, 2017, to December 31, 2021 (Table 1). Among 50 patients, sixteen were male and the ratio of male to female was $1: 2$. The average age at the time of diagnosis was $47.4 \pm 11.8$ years (range, $18-76$ years). Only 3 patients were current smokers. Family screening was available in 11 of 31 identified families.

\section{Clinical Features}

Spontaneous pneumothorax had occurred in 27 patients; the characteristics of pneumothorax was described in 26 cases in detail, including the age at onset of pneumothorax, number of episodes, and side of pneumothorax. Fifty-four symptomatic episodes of pneumothorax had occurred; the mean number of pneumothoraces was $2.1 \pm 1.5$ (range, 1 to 7 ). Among these pneumothoraces, there were 3 episodes of bilateral pneumothorax. Family history of pneumothorax was identified in $58 \%$ of the families.

Forty-eight patients manifested pulmonary cysts on CT (one refusing the CT scans) (Table 2). The cystic lesions were located bilaterally in 47 cases (98\%) and all manifested lower lung predominance with irregular shapes. Pulmonary cysts were detected only unilaterally in a thirty-year-old male. In most cases, the maximum diameters of cysts ranged $1-5 \mathrm{~cm}$; the largest cystic lesion measured $8.5 \mathrm{~cm}$. The number of cysts was $>20$ in 90\% (43/48) patients and 85\% (41/48) presented paramediastinal (medial) cysts. Lung adenocarcinoma and pulmonary sclerosing pneumocytoma was discovered in two patients, respectively, from the same family and another patient also had a pulmonary adenocarcinoma.

Skin lesions were found in 32/47 (68\%) patients and were characterized by skin-colored papules on faces and cervico-thoracic regions. Nine patients had undergone biopsy which showed fibrofolliculomas in 2 , trichodiscoma in 1, and epidermoid cysts in 6 cases. The mean ages were $50.9 \pm 9.8$ and $37.4 \pm 9.6$ in patients with skin lesions and those with normal skin, respectively. 
Renal tumors were detected in only two (4\%) patients. One patient underwent a partial nephrectomy which showed renal chromophobe cell carcinoma at the age of 64 years. The second patient was diagnosed to have bilateral renal cancer on magnetic resonance imaging (MRI) and is undergoing followup observation. In addition, renal angiomyolipoma (AML) was detected in four patients, a type of kidney tumor that is not usually observed in patients with BHDS and is characteristically associated with tuberous sclerosis complex (TSC).

Pathogenic variants in the $F L C N$ gene were detected in 44 patients. The most frequent type $(23 \%, 10 / 44)$ was the single deletion, duplication of cytosine in codon 1285 of exon 11 (c.1285del, c.1285dup), follow by the mutation of c.1579_1580ins in exon 14 and exon 9 (c.1015C囚T) (16\%, 7/44). Other mutations occurred much less frequently. Mutation c. $1015 \mathrm{C} \otimes \mathrm{T}$ was associated with a $29 \%$ risk (2 of 7 patients) for pneumothorax, and $50 \%$ for mutation c. $1285 \mathrm{dup} / \mathrm{del}$ ( 5 of 10 patients), and $57 \%$ for mutation c.1579_1580ins (4 of 7 patients). Mutation c.1015C $\triangle \mathrm{T}$ was associated with a 57\% risk (4 of 7 patients) for skin lesions, which increased to $71 \%$ for mutation c.1579_1580ins (5 of 7 patients), and $90 \%$ for mutation c.1285del/dup (9 of 10 patients). However, the differences in the risk of pneumothorax ( $P$ value, $0.67)$ and skin lesions ( $P$ value, 0.35$)$ associated with these three most common mutations were not statistically significant. One patient with renal carcinoma manifested mutation c.1429C $₫ \mathrm{~T}$ in exon 12 , the other refused genetic testing while her daughter carried mutation c.1381_1382insA.

\section{Diagnoses and managements}

After the first case in 2017, there were 1, 8, 8 and 13 families with BHDS newly diagnosed from 2018 to 2021, respectively. Except for the first 2 patients, the remainder were diagnosed after the start of our Rare Lung Disease Clinic in June 2019. Forty-four patients were confirmed by direct sequencing of the FLCN gene and the remaining six by clinical diagnosis. Twenty-one (42\%) patients were suspected due to pneumothorax and $18(36 \%)$ cases due to family screening. The remaining $10(20 \%)$ cases were diagnosed by radiologic findings of diffuse pulmonary cysts except for one patient who manifested a renal mass. Forty-four patients were confirmed to have BHDS by detection of pathogenic variants in the FLCN gene; others were diagnosed by clinical features. Almost all initial presentations involved pneumothorax, which was often misdiagnosed as primary spontaneous pneumothorax without the appreciation of the underlying hereditary disorder. The first case of BHDS had been misdiagnosed as pulmonary lymphangioleiomyomatosis (LAM). The average interval from initial presentation to eventual diagnosis of BHDS was $8.3 \pm 9.1$ years (range from 1 to 44 years). The correct diagnosis was delayed 4.7 years for patients without pneumothorax history.

Twenty-four patients with pneumothorax received surgical treatment and pneumothorax recurred in only one case during the follow-up period. Among them, 8 patients with pneumothorax were treated by bullectomy with pleurodesis, 5 by bullectomy alone; the information on the type of surgical procedure was not available in the remaining 11 patients. The recurrence rate of pneumothorax after conservative treatment (including tube thoracostomy) was $22 / 29$ (76\%) while the pneumothorax recurred after surgical treatment (bullectomy and/or pleurodesis) in only $1 / 24(4 \%)$. Partial nephrectomies were performed in 
one renal cancer patient and another patient refused the renal operation. All three cases with pulmonary tumors underwent lobectomy.

Fifteen patients underwent annual follow-up visits in our hospital. The average duration of follow-up was 17 months per patient (range, 4 to 36 months). Spontaneous pneumothorax was detected on to chest computed tomography in a patient at her 18-months-follow-up visit and was initially managed conservatively. After six-month observation, a large pneumothorax recurred on the same side, and she underwent bullectomy with pleurodesis. No new kidney lesions were detected during the follow-up period.

\section{Discussion}

Striking progress has been achieved with respect to the diagnosis and management of rare diseases in China in recent decades. Although more than 220 cases with BHDS has been reported up to December 2020 in China(7), these cases were distributed mostly in the Beijing and Jiangsu Province due to advanced medical resources and great economic levels associated with these regions $(7,19)$. To our knowledge, current study describes the largest cohort of patients with BHDS in the Chinese population. The number of patients and families diagnosed in our hospital has increased year by year, especially after the initiation of our Rare Lung Disease Clinic.

Multiple pulmonary cysts detected on chest CT are the most common manifestation and has been described in $85-99 \%$ of cases in various study cohorts $(7,8,10)$. The number, shape, size, and distribution of pulmonary cystic lesions are helpful to distinguish BHDS from other cystic lung diseases $(20,21)$. On chest CT, BHDS is characterized by multiple, irregular-shaped cysts of varying sizes with medial and basal predominance in distribution $(20,22,23)$. These features were observed in most of the patients in our study. The detection of lung cysts led to the diagnosis of BHDS in one-fifth of our cases with a relatively shorter delay to eventual diagnosis ( 4.7 years) compared to those with other presentation modes.

The presence of multiple pulmonary cysts is associated with spontaneous pneumothorax which is 50times more likely to occur in BHD-affected individuals compared to those not affected as found by Zbar and colleagues(24). In 2008, Ren et al first demonstrated that FLCN mutation contributes to not only familial primary spontaneous pneumothorax but also patients with apparently sporadic (nonfamilial) primary spontaneous pneumothorax(6). Recent studies showed $64 \%$ of familial spontaneous pneumothorax was BHDS in China and $6 / 7$ in Italy(19). Thus, $42 \%$ of our patients were suspected due to pneumothorax history. Less than $60 \%$ of patients had experienced at least one episode of spontaneous pneumothorax and had family history of pneumothorax in our study, which are lower than previous data in China ( $71 \%$ and $85 \%$, respectively)(7). These differences might reflect the earlier recognition of BHDS prior to onset of pneumothoraces.

In our cohort, BHDS-related pneumothorax was associated with a higher recurrence rate after conservative therapy compared to surgical intervention. Similar finding was observed for a thoracic surgery cohort reported from Beijing and a patient survey study in America $(8,19)$. The high recurrence 
rate after conservative management may be due to the multiplicity of pulmonary cysts in patients with BHD syndrome, which predispose to recurrent pneumothoraces. Surgical pleurodesis (mechanical abrasion) was recommended in the American Thoracic Society/Japanese Respiratory Society Clinical Practice Guideline to manage pneumothorax in another diffuse cystic lung disease, LAM(25). Thus, early surgical management should be considered for patients presenting with BHDS-related pneumothorax.

Skin lesions in patients with BHDS usually appear after the age of 20 years, as multiple, dome-shaped, whitish papules on the face, neck, and sometimes trunk(26). Skin lesions are a common feature observed in Caucasian patients with BHDS, in whom it is found in over $90 \%$ (26). However, the patients in China seem to have a lower prevalence of skin lesions (18\%) (7). These finding was also reported in other East Asian countries $(10,27)$. However, our study shows that skin lesions were present in nearly $70 \%$ of patients and suggests this feature may have been overlooked previously. This explanation is supported by the observation that the prevalence of skin manifestations in patients with BHDS reported from Peking Union Medical College hospital (PUMCH) increased from 11\% in 2017 to $47.2 \%$ in 2019 and $54.5 \%$ in $2020(11,17,20)$. Furthermore, Iwabuchi et al detected skin lesions in 26/31 (83.9\%) Japanese patients with BHDS using dermoscopy and skin biopsy confirmed fibrofolliculoma and/or trichodiscoma histologically in $73.9 \%(28)$. Skin biopsy in the context of an experienced multidisciplinary team is helpful to recognize skin findings suspicious for BHDS. Thus, recent evidence suggests that the skin involvement in BHDS may not be significantly lower in Chinese patients compared to Caucasians.

Renal cancer is the most serious complication of BHDS, are often bilateral and multifocal, with the oncocytoma and chromophobe variant being the most common types of malignancy(29). The risk of developing renal tumors is seven-fold higher in patients with BHDS, compared to the general population(24). The low prevalence of renal involvement in our cohort may be due to selection bias since nearly all patients in our study were recruited from respiratory department. Another reason might be lack of long-term follow-up for most patients. However, further studies on potential racial differences int the prevalence of renal cancer may be warranted.

The most frequent mutations associated with BHDS in our study were c.1285del/dup on exon 11, similar to the results in previous studies $(30,31)$. Toro et al. reported that $48 \%$ mutations were identified in exon 11 , as a mutation 'hotspot' for BHDS(31). The most frequent mutation in Chinese BHDS patients was the single deletion, duplication of cytosine in codon 1285 of exon 11 as reported from PUMCH (11). Mutation c. $1015 \mathrm{C}>\mathrm{T}$ accounted for about $1 / 6$ of patients in this study and three novel mutations of c.1381_1382insA in exon 12, c.761TC in exon 7, c.1283_1284insA in exon 11 were encountered. Mutation was not detected in a forty-three-year-old female who manifested fibrofolliculomas by skin biopsy. Toro et al. reported that patients with mutation in exon 9 or 12 have a higher risk of pneumothorax compared to those patients carrying mutation in other exons(31). A recent study from Germany reported that the mutation c.250-2A > G was associated with twice as high risk of pneumothorax compared with the mutation c.1285dup (30). However, the correlation between genotype and phenotype was not found in our study. This issue needs to be explored further with larger number of patients. 
It was reported that 399 patients with BHDS were identified in Netherlands within the total population of about 17.1 million (32). Anhui Province, located in Eastern of China with 61-million citizens, would be estimated to have over 1400 estimated patients with BHDS. There has been no previous data regarding the actual prevalence of BHDS in China. Although our study cohort in Anhui Province comprises the largest number of cases of BHDS described from China, it only accounts for $3.6 \%$ of estimated total number of cases in our Province. This highlights the underrecognition of rare diseases in developing regions of the world. Establishment of a Rare Lung Disease Clinic supported multidisciplinary team at our medical center led to improved recognition and diagnosis of BHDS over a 2.5-year period. Similar model of care may allow better care of patients with rare diseases in other developing parts of the world.

There were several limitations to our study. Firstly, the number of cases is modest in this cohort. However, considering the rarity of disease we have collected a relatively large cohort in a single center. Secondly, due to the retrospective nature of this study, the family history/screening and annual follow-up evaluations were not always available. Thirdly, skin biopsies were only performed on 9 patients as many individuals refused to undergo this procedure. However, all the skin lesions were examined by a same multidisciplinary team focused on diffuse cystic lung diseases. A long-term, muti-center prospective study is needed to allow better understanding of this rare disease that will improve management.

\section{Conclusion}

There has been increasing number of patients with BHDS recognized in China, facilitated by establishment of a Rare Lung Disease Clinic. Pulmonary cysts and pneumothorax were commonly encountered features, but skin lesions appeared to be more prevalent in Chinese subjects than previously reported in other Asian countries.

\section{Declarations}

\section{Acknowledgments}

We thank all the patients and families for their contribution to this work. We also want to thank Prof. Yaping Liu, Chinese Academy of Medical Sciences, for her contribution in genetic analysis of FLCN mutations.

\section{Author contributions}

X.H. and G.Z. designed the study and drafted the manuscript. B.S. and L.Y. performed the molecular genetic testing, mutation analysis and manuscript editing. X.H., G.Z., G.F., Y.W. and Y.W. collected patients' samples and clinical information, contributed to data analysis. J.L. was consulted with patients' skin lesions and performed the histological analysis of biopsied specimens. W.W. read all the CT scans of BHDS and assess all the CT features of BHDS patients. X.J. performed pneumothorax surgery and manuscript editing. J.X. performed renal surgery and manuscript editing. Y.P. reviewed all the pathological 
slides and checked the manuscript. J.H. R supervised this study, checked, and revised this manuscript. All the authors reviewed and approved the final version of the manuscript.

\section{Funding}

Key medical and health specialty construction project of Anhui Province

\section{Availability of data and materials}

The datasets generated and analyzed for this study are not publicly available due to participant privacy but are available from the corresponding author upon reasonable request.

\section{Ethics approval and consent to participate}

This study was approved by the Ethics Committee of the First Affiliated Hospital of University of Science and Technology of China (Number 2021 KY187). All subjects included in this study signed informed consent documents.

\section{Consent for publication}

Consent for publication was obtained from all participants.

\section{Competing interests}

No competing interests were declared.

\section{Author details}

${ }^{1}$ Department of Pulmonary and Critical Care Medicine, the First Affiliated Hospital of USTC, Division of Life Sciences and Medicine, University of Science and Technology of China, Hefei, Anhui, China. ${ }^{2}$ WanNan Medical College, Wuhu, Anhui, China. ${ }^{3}$ Department of Dermatology, the First Affiliated Hospital of USTC, Division of Life Sciences and Medicine, University of Science and Technology of China, Hefei, Anhui, China. ${ }^{4}$ BengBu Medical College, Bengbu, Anhui, China. ${ }^{5}$ Department of Thoracic Surgery, the First Affiliated Hospital of USTC, Division of Life Sciences and Medicine, University of Science and Technology of China, Hefei, Anhui, China. ${ }^{6}$ Department of Pathology, the First Affiliated Hospital of USTC, Division of Life Sciences and Medicine, University of Science and Technology of China, Hefei, Anhui, China. ${ }^{7}$ Department of Urology, the First Affiliated Hospital of USTC, Division of Life Sciences and Medicine, University of Science and Technology of China, Hefei, Anhui, China. ${ }^{8}$ Department of Radiology, the First Affiliated Hospital of USTC, Division of Life Sciences and Medicine, University of Science and Technology of China, Hefei, Anhui, China. ${ }^{9}$ School of Basic Medicine, Anhui Medical University. ${ }^{10}$ Jiangsu Key Laboratory of Molecular Medicine, School of Medicine, Nanjing University, Nanjing, Jiangsu, China. ${ }^{11}$ Division of Pulmonary and Critical Care Medicine, Mayo Clinic, Rochester, MN. 


\section{References}

1. Schmidt LS, Warren MB, Nickerson ML, Weirich G, Matrosova V, Toro JR, et al. Birt-Hogg-Dubé syndrome, a genodermatosis associated with spontaneous pneumothorax and kidney neoplasia, maps to chromosome 17p11.2. Am J Hum Genet. 2001;69(4):876-82.

2. Gupta N, Sunwoo BY, Kotloff RM. Birt-Hogg-Dubé syndrome. Clin Chest Med. 2016;37(3):475-86.

3. Hornstein OP, Knickenberg M. Perifollicular fibromatosis cutis with polyps of the colon-a cutaneointestinal syndrome sui generis. Arch Dermatol Res. 1975;253(2):161-75.

4. Birt AR, Hogg GR, Dubé WJ. Hereditary multiple fibrofolliculomas with trichodiscomas and acrochordons. Arch Dermatol. 1977;113(12):1674-7.

5. Muller ME, Daccord C, Taffe P, Lazor R. Prevalence of Birt-Hogg-Dubé syndrome determined through epidemiological data on spontaneous pneumothorax and Bayes theorem. Front Med (Lausanne). 2021;8:631168.

6. Ren HZ, Zhu CC, Yang C, Chen SL, Xie J, Hou YY, et al. Mutation analysis of the FLCN gene in Chinese patients with sporadic and familial isolated primary spontaneous pneumothorax. Clin Genet. 2008;74(2):178-83.

7. Hu X, Zhang G, Chen X, Xu KF. Birt-Hogg-Dubé syndrome in Chinese patients: a literature review of 120 families. Orphanet J Rare Dis. 2021;16(1):223.

8. Gupta N, Kopras EJ, Henske EP, James LE, El-Chemaly S, Veeraraghavan S, et al. Spontaneous pneumothoraces in patients with Birt-Hogg-Dubé syndrome. Ann Am Thorac Soc. 2017;14(5):70613.

9. Guo T, Shen Q, Ouyang R, Song M, Zong D, Shi Z, et al. The clinical characteristics of East Asian patients with Birt-Hogg-Dubé syndrome. Ann Transl Med. 2020;8(21):1436.

10. Furuya M, Yao M, Tanaka R, Nagashima Y, Kuroda N, Hasumi H, et al. Genetic, epidemiologic and clinicopathologic studies of Japanese Asian patients with Birt-Hogg-Dubé syndrome. Clin Genet. 2016;90(5):403-12.

11. Liu K, Xu W, Tian X, Xiao M, Zhao X, Zhang Q, et al. Genotypic characteristics of Chinese patients with BHD syndrome and functional analysis of FLCN variants. Orphanet J Rare Dis. 2019;14(1):223.

12. Sun W, Gao M, Fei G, Wei W, Hu X. Case report of recurrent pneumothorax with underlying diffuse lung cysts. Chin J Lung Dis(Electronic Edition). 2019;12(2):241-3. (in Chinese).

13. Lee JE, Cha YK, Kim JS, Choi JH. Birt-Hogg-Dubé syndrome: Characteristic CT findings differentiating it from other diffuse cystic lung diseases. Diagn Interv Radiol. 2017;23(5):354-9.

14. Ebana H, Mizobuchi T, Kurihara M, Kobayashi E, Haga T, Okamoto S, et al. Novel clinical scoring system to identify patients with pneumothorax with suspicion for Birt-Hogg-Dubé syndrome. Respirology. 2018;23(4):414-8.

15. Fabre A, Borie R, Debray MP, Crestani B, Danel C. Distinguishing the histological and radiological features of cystic lung disease in Birt-Hogg-Dubé syndrome from those of tobacco-related spontaneous pneumothorax. Histopathology. 2014;64(5):741-9. 
16. Menko FH, van Steensel MA, Giraud S, Friis-Hansen L, Richard S, Ungari S, et al. Birt-Hogg-Dubé syndrome: Diagnosis and management. Lancet Oncol. 2009;10(12):1199-206.

17. Liu Y, Xu Z, Feng R, Zhan Y, Wang J, Li G, et al. Clinical and genetic characteristics of chinese patients with Birt-Hogg-Dubé syndrome. Orphanet J Rare Dis. 2017;12(1):104.

18. Cai M, Zhang X, Fan L, Cheng S, Kiram A, Cen S, et al. A novel FLCN intragenic deletion identified by NGS in a BHDS family and literature review. Front Genet. 2021;12:636900.

19. Liu Y, Xing H, Huang Y, Meng S, Wang J. Familial spontaneous pneumothorax: Importance of screening for Birt-Hogg-Dubé syndrome. Eur J Cardiothorac Surg. 2020;57(1):39-45.

20. Xu W, Xu Z, Liu Y, Zhan Y, Sui X, Feng R, et al. Characterization of CT scans of patients with BirtHogg-Dubé syndrome compared with those of Chinese patients with non-BHD diffuse cyst lung diseases. Orphanet J Rare Dis. 2020;15(1):176.

21. Lee E, Sayyouh M, Haggerty JE, Kazerooni E, Agarwal PP. Role of radiologists in the diagnosis of unsuspected Birt-Hogg-Dubé syndrome in a tertiary clinical practice. AJR Am J Roentgenol. 2019;213(4):792-7.

22. Tobino K, Gunji Y, Kurihara M, Kunogi M, Koike K, Tomiyama N, et al. Characteristics of pulmonary cysts in Birt-Hogg-Dubé syndrome: Thin-section CT findings of the chest in 12 patients. Eur J Radiol. $2011 ; 77(3): 403-9$.

23. Escalon JG, Richards JC, Koelsch T, Downey GP, Lynch DA. Isolated cystic lung disease: An algorithmic approach to distinguishing Birt-Hogg-Dubé syndrome, Lymphangioleiomyomatosis, and Lymphocytic Interstitial Pneumonia. Am J Roentgenol. 2019:1-5.

24. Zbar B, Alvord WG, Glenn G, Turner M, Pavlovich CP, Schmidt L, et al. Risk of renal and colonic neoplasms and spontaneous pneumothorax in the Birt-Hogg-Dubé syndrome. Cancer Epidemiol Biomarkers Prev. 2002;11(4):393-400.

25. Gupta N, Finlay GA, Kotloff RM, Strange C, Wilson KC, Young LR, et al. Lymphangioleiomyomatosis diagnosis and management: High-Resolution chest Computed Tomography, transbronchial lung biopsy, and pleural disease management. Am J Respir Crit Care Med. 2017;196(10):1337-48.

26. Steinlein OK, Ertl-Wagner B, Ruzicka T, Sattler EC. Birt-Hogg-Dubé syndrome: An underdiagnosed genetic tumor syndrome. J Dtsch Dermatol Ges. 2018;16(3):278-83.

27. Lee JH, Jeon MJ, Song JS, Chae EJ, Choi JH, Kim GH, et al. Birt-Hogg-Dubé syndrome in Korean: Clinicoradiologic features and long term follow-up. Korean J Intern Med. 2019;34(4):830-40.

28. Iwabuchi C, Ebana H, Ishiko A, Negishi A, Mizobuchi T, Kumasaka T, et al. Skin lesions of Birt-HoggDubé syndrome: Clinical and histopathological findings in 31 Japanese patients who presented with pneumothorax and/or multiple lung cysts. J Dermatol Sci. 2018;89(1):77-84.

29. Furuya M, Hasumi H, Yao M, Nagashima Y. Birt-Hogg-Dubé syndrome-associated renal cell carcinoma: Histopathological features and diagnostic conundrum. Cancer Sci. 2020;111(1):15-22.

30. Sattler EC, Syunyaeva Z, Mansmann U, Steinlein OK. Genetic risk factors for spontaneous pneumothorax in Birt-Hogg-Dubé syndrome. Chest. 2020;157(5):1199-206. 
31. Toro JR, Wei MH, Glenn GM, Weinreich M, Toure O, Vocke C, et al. BHD mutations, clinical and molecular genetic investigations of Birt-Hogg-Dubé syndrome: A new series of 50 families and a review of published reports. J Med Genet. 2008;45(6):321-31.

32. van de Beek I, Glykofridis IE, Wolthuis RMF, Gille H, Johannesma PC, Meijers-Heijboer HEJ, et al. No evidence for increased prevalence of colorectal carcinoma in 399 Dutch patients with Birt-Hogg-Dubé syndrome. Br J Cancer. 2020;122(4):590-4.

\section{Tables}

Table 1 Clinical characteristics of study population

\begin{tabular}{ll} 
Characteristics & $(\mathrm{n}=50)$ \\
\hline Male/Female & $16 / 34$ \\
\hline age at examination- $y r$ & $47.3 \pm 11.7(18-76)$ \\
\hline & \\
\hline Pulmonary manifestations & $(\mathrm{n}=49)$ \\
\hline age at onset of pneumothorax- $\mathrm{yr}$ & $41.9 \pm 10.9(20-62)$ \\
\hline cysts on chest CT & $48 / 49(98 \%)$ \\
\hline pneumothorax & $27 / 50(54 \%)$ \\
\hline & \\
\hline Skin manifestations & $(\mathrm{n}=47)$ \\
\hline multiple skin-colored papules & $32 / 47(68 \%)$ \\
\hline FFs and/or TDs confirmed & $3 / 47(6 \%)$ \\
\hline & \\
\hline Renal manifestations & $(\mathrm{n}=41)$ \\
\hline cancer & $2 / 41(5 \%)$ \\
\hline AML & $4 / 41(10 \%)$ \\
\hline
\end{tabular}

Family history of pneumothorax $\quad 29 / 50(58 \%)$

\begin{tabular}{ll}
\hline FLCN Genetic testing & $(\mathrm{n}=45)$ \\
\hline germline mutation & $44 / 45(98 \%)$
\end{tabular}


Age was presented as mean $\pm S D$ (range).

Table 2 The characteristics of pulmonary cysts observed on CT

\begin{tabular}{ll} 
Feature & cases(n=48) \\
\hline Number & $2(4 \%)$ \\
\hline$>10$ & $3(6 \%)$ \\
\hline $10-20$ & $43(90 \%)$ \\
\hline$<20$ & \\
\hline Distribution & \\
\hline Bilateral to unilateral & $47(98 \%)$ \\
\hline Bilateral & $1(2 \%)$ \\
\hline Unilateral & \\
\hline Upper to lower & 0 \\
\hline Upper dominant & $48(100 \%)$ \\
\hline Lower dominant & \\
\hline Central to peripheral & $41(85 \%)$ \\
\hline Central dominant & $14(29 \%)$ \\
\hline Peripheral dominant & $4(8 \%)$ \\
\hline Neither Central nor peripheral & \\
\hline Size of maximum cysts & $10(21 \%)$ \\
\hline$<1 \mathrm{~cm}$ & $9(19 \%)$ \\
\hline $1-2 \mathrm{~cm}$ & $27(56 \%)$ \\
\hline $2-5 \mathrm{~cm}$ & \\
\hline$>5 \mathrm{~cm}$ & \\
\hline Shape & \\
\hline Round to oval & \\
\hline Irregular & \\
\hline
\end{tabular}

Table 3 The prevalence of skin lesions in different regions of the world 


\begin{tabular}{lllll} 
Author & Years & Region & Cases & The prevalence of skin lesions \\
\hline Laura S. Schmidt et al.+ & 2005 & Europe & 219 & $84 \%$ \\
\hline Nishant Gupta et al.\$ & 2017 & America & 104 & $71 \%$ \\
\hline Joo Hee Lee et al.\& & 2019 & Korea & 12 & $50 \%$ \\
\hline Mitsuko Furuya et al.\# & 2016 & Japan & 156 & $49 \%$ \\
\hline Keqiang Liu et al.* & 2019 & Beijing & 39 & $47 \%$ \\
\hline This study & 2021 & Anhui & 47 & $68 \%$
\end{tabular}

+data from Am. J. Hum. Genet 2005,76:1023-1033; \$data from Ann Am Thorac Soc 2017,14 (5): 706713; \&data from Korean J Intern Med 2019,34(4):830-840; \#data from Clin Genet 2016, 90: 403-412; *data from Orphanet J Rare Dis 2019,15;14(1):223

\section{Figures}

0

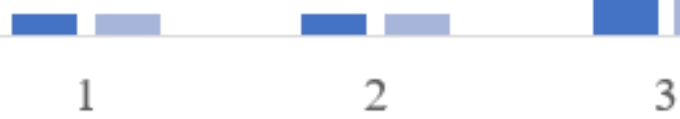

3

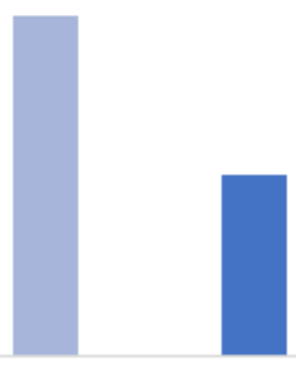

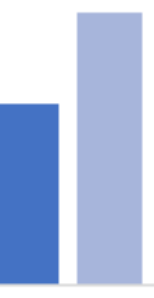

4

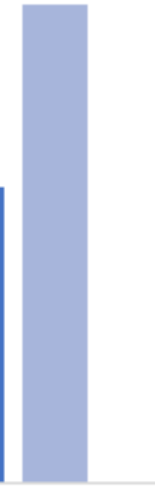

5

\section{Figure 1}

Number of families and cases of BHDS distributed by year of diagnosis 


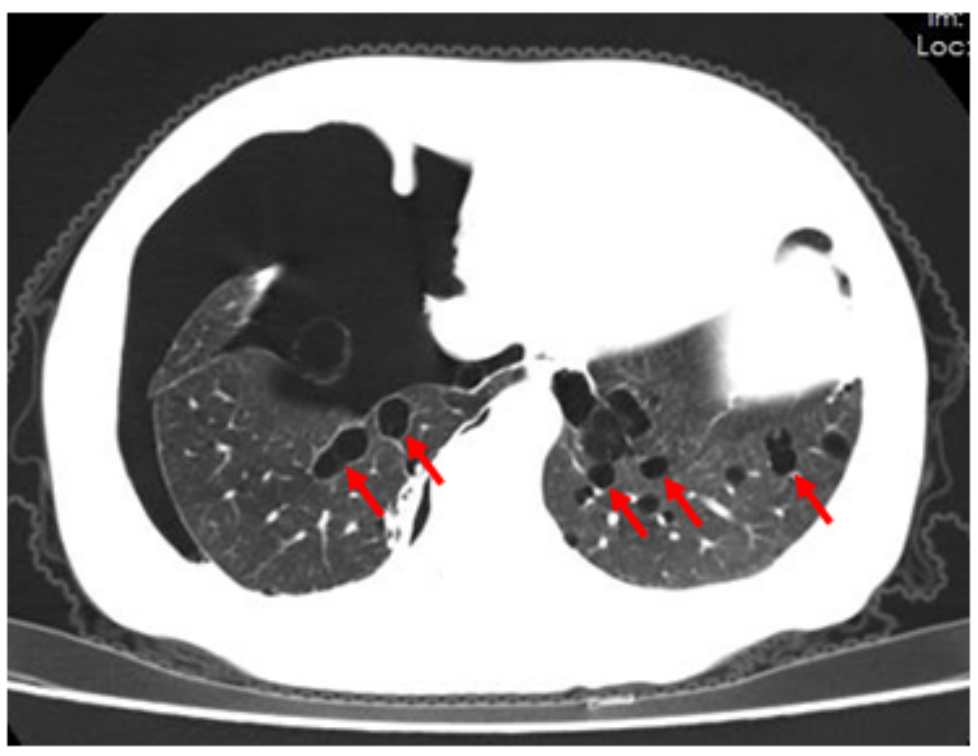

Figure 2

BHD syndrome in a 54-year-old female. Chest CT image shows multiple cysts of varying sizes and irregular shapes predominantly in the lower lungs, and pneumothorax on the right side. 


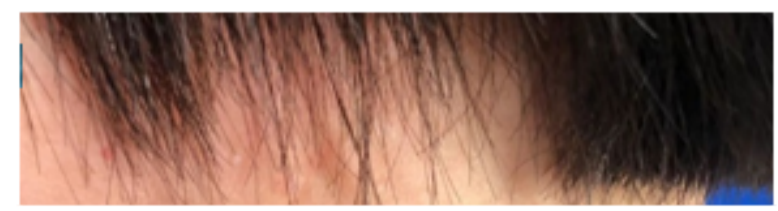

\section{Figure 3}

Skin lesions of BHD syndrome observed in a 48-year-old female. A: Multiple pale and dome-shaped papules on the patient's neck. B: Hematoxylin and eosin staining of skin biopsy showed histologic features consistent with trichodiscoma $(\times 200)$.

\section{Supplementary Files}

This is a list of supplementary files associated with this preprint. Click to download.

- Appendix.docx 